\title{
Thermal Transport in Crystals as a Kinetic Theory of Relaxons
}

\author{
Andrea Cepellotti and Nicola Marzari* \\ Theory and Simulations of Materials (THEOS), and National Centre for Computational Design \\ and Discovery of Novel Materials (MARVEL), École Polytechnique Fédérale de Lausanne, \\ Station 9, 1015 Lausanne, Switzerland
}

(Received 25 April 2016; published 17 October 2016)

\begin{abstract}
Thermal conductivity in dielectric crystals is the result of the relaxation of lattice vibrations described by the phonon Boltzmann transport equation. Remarkably, an exact microscopic definition of the heat carriers and their relaxation times is still missing: Phonons, typically regarded as the relevant excitations for thermal transport, cannot be identified as the heat carriers when most scattering events conserve momentum and do not dissipate heat flux. This is the case for two-dimensional or layered materials at room temperature, or three-dimensional crystals at cryogenic temperatures. In this work, we show that the eigenvectors of the scattering matrix in the Boltzmann equation define collective phonon excitations, which are termed here "relaxons". These excitations have well-defined relaxation times, directly related to heat-flux dissipation, and they provide an exact description of thermal transport as a kinetic theory of the relaxon gas. We show why Matthiessen's rule is violated, and we construct a procedure for obtaining the mean free paths and relaxation times of the relaxons. These considerations are general and would also apply to other semiclassical transport models, such as the electronic Boltzmann equation. For heat transport, they remain relevant even in conventional crystals like silicon, but they are of the utmost importance in the case of twodimensional materials, where they can revise, by several orders of magnitude, the relevant time and length scales for thermal transport in the hydrodynamic regime.
\end{abstract}

DOI: 10.1103/PhysRevX.6.041013

\section{INTRODUCTION}

The foundations for the theories of lattice thermal transport were set in place long ago, from the phonon Boltzmann transport equation (BTE) [1] to Green-Kubo linear-response theory $[2,3]$. However, only recently has it become possible, thanks to our increased computational capabilities, to solve these transport models with high accuracy and without resorting to oversimplifying assumptions [4-15].

In particular, the linear BTE can nowadays be solved exactly, using empirical or first-principles interactions, with iterative $[4,16,17]$, variational $[7,18-20]$, or direct diagonalization algorithms [21-23], none of which needs to simplify the scattering operator with the often-adopted single-mode relaxation time approximation (SMA). In the SMA, each phonon mode relaxes independently to equilibrium, and it has long been known that this is an incorrect assumption for solids at low temperatures [18,24]. Importantly, this approximation fails dramatically in lower

*nicola.marzari@epfl.ch

Published by the American Physical Society under the terms of the Creative Commons Attribution 3.0 License. Further distribution of this work must maintain attribution to the author(s) and the published article's title, journal citation, and DOI.
Subject Areas: Computational Physics,

Condensed Matter Physics,

Materials Science dimensions, as first found in graphene [25], boron nitride, and other two-dimensional (2D) materials [26-28], as well as in layered crystals [29]. The origin of such failure of the SMA has been, up to now, a matter of debate, with an emerging picture of collective phonon excitations being responsible for heat transfer [26,29-33], while nevertheless lacking a definition of such excitations.

The microscopic interpretation of thermal transport in the BTE is based on the kinetic theory of gases, used in various contexts since its development in the 19th century, which relates the thermal conductivity to the velocities and relaxation times of the carriers, with phonons usually being identified as the relevant gas of excitations. However, as argued below, this identification is incorrect since only the adoption of the SMA allows for the definition of a time interval (i.e., a lifetime) between heat-flux dissipation events taken as phonon scatterings. Going beyond the SMA, the full, exact solution of the BTE provides the correct thermal conductivity (dramatically improving predictions in 2D materials or at low temperatures) but adds complexity in its interpretation. In fact, exactly solving the BTE implies abandoning the concept of phonon relaxation time and the description of heat being carried by a gas of phonons. In other words, phonon lifetimes or phonon mean free paths are no longer relevant quantities to describe thermal transport since phonons are not the heat carriers 
anymore. Yet, the beauty of the SMA description lies in the simplicity of its description of transport. A natural question then arises: Can one define heat carriers, relaxation times, or mean free paths within the exact treatment of the BTE?

In this work, we provide an answer to these questions. First, we recall why phonon lifetimes are unrelated to heat-flux dissipation. Then, we define a set of collective excitations, termed relaxons, that diagonalize the scattering matrix. The BTE is rewritten in the basis of these relaxons; in this representation, each eigenvector represents a collective excitation consisting of a linear combination of out-of-equilibrium phonon populations, and it describes the thermal relaxation of a collective excitation of out-ofequilibrium lattice vibrations. We show that each relaxon is characterized by a well-defined relaxation time; in the case of a homogeneous system, at the steady state, each relaxon also has a well-defined velocity and mean free path, and the thermal conductivity can be interpreted exactly as a kinetic theory of the relaxon gas. As a practical example, we compare thermal conductivities in graphene and silicon, contrasting the relaxon and the phonon representations, and highlight the profoundly different pictures that emerge.

\section{APPROXIMATED RELAXATION TIMES}

We start our derivation by recalling the microscopic description of heat transport given by the linearized phonon BTE [18]:

$$
\frac{\partial n_{\mu}(\boldsymbol{x}, t)}{\partial t}+\boldsymbol{v}_{\mu} \cdot \nabla n_{\mu}(\boldsymbol{x}, t)=-\frac{1}{\mathcal{V}} \sum_{\mu^{\prime}} \Omega_{\mu \mu^{\prime}} \Delta n_{\mu^{\prime}}(\boldsymbol{x}, t) .
$$

This equation describes the out-of-equilibrium dynamics of the phonon excitation number $n_{\mu}$ at position $\boldsymbol{x}$ and time $t$, for all possible phonon states $\mu$ (in shorthand notation, $\mu \equiv(\boldsymbol{q}, s)$, where $\boldsymbol{q}$ varies over the Brillouin-zone and $s$ over the phonon branches). Furthermore, $v_{\mu}$ is the phonon group velocity, $\mathcal{V}$ is a normalization volume, $\Omega_{\mu \mu^{\prime}}$ is the linear phonon scattering operator, and $\Delta n_{\mu}=n_{\mu}-\bar{n}_{\mu}$ is the deviation of the phonon distribution from thermal equilibrium, i.e., the Bose-Einstein distribution $\bar{n}_{\mu}(\boldsymbol{x}, t)=$ $\left(e^{\hbar \omega_{\mu} / k_{B} T(\boldsymbol{x}, t)}-1\right)^{-1}$, with $\omega_{\mu}$ being the phonon frequency and $T(\boldsymbol{x}, t)$ the local temperature. This linear approximation, commonly used in most studies of transport, allows one to describe scattering as a linear operator represented by the action of the matrix $\Omega_{\mu \mu^{\prime}}$ on $\Delta n_{\mu}$ : This assumption holds for small deviations from thermal equilibrium and will always be used in the rest of the manuscript. The scattering matrix appearing in Eq. (1) is in its most general form and describes all possible mechanisms by which a phonon excitation can be transferred from a state $\mu$ to a state $\mu^{\prime}$. For the sake of simplicity, here we limit ourselves to the inclusion of three-phonon processes and isotopic scattering events [7], whose expressions are reported for completeness in Appendix A.
For later convenience, it is useful to write the left-hand side of Eq. (1) in terms of the unknown $\Delta n_{\mu}$ :

$$
\begin{aligned}
& \frac{\partial \bar{n}_{\mu}}{\partial T}\left(\frac{\partial T(\boldsymbol{x}, t)}{\partial t}+\boldsymbol{v}_{\mu} \cdot \nabla T(\boldsymbol{x}, t)\right)+\frac{\partial\left(\Delta n_{\mu}(\boldsymbol{x}, t)\right)}{\partial t} \\
& \quad+\boldsymbol{v}_{\mu} \cdot \nabla\left(\Delta n_{\mu}(\boldsymbol{x}, t)\right) \\
& \quad=-\frac{1}{\mathcal{V}} \sum_{\mu^{\prime}} \Omega_{\mu \mu^{\prime}} \Delta n_{\mu^{\prime}}(\boldsymbol{x}, t),
\end{aligned}
$$

where $T$ is the reference temperature at which the BTE has been linearized. To obtain Eq. (2), we substituted $n_{\mu}=$ $\bar{n}_{\mu}+\Delta n_{\mu}$ in Eq. (1) and used the fact that the Bose-Einstein distribution depends on space and time only through the temperature $T(\boldsymbol{x}, t)$.

A closed-form solution of the above equation can be obtained in the SMA, which replaces the scattering operator with its diagonal terms

$$
\frac{1}{\mathcal{V}} \sum_{\mu^{\prime}} \Omega_{\mu \mu^{\prime}} \Delta n_{\mu^{\prime}}(\boldsymbol{x}, t) \approx \frac{\Delta n_{\mu}(\boldsymbol{x}, t)}{\tau_{\mu}^{\mathrm{SMA}}} .
$$

To show that, in this simplified diagonal form, $\tau_{\mu}^{\text {SMA }}$ indeed represents a relaxation time, let us consider a system at thermal equilibrium $[T(\boldsymbol{x}, t)=T]$ so that the phonon distribution is $\bar{n}_{\mu}$ everywhere; thus, in Eq. (2), $\nabla\left(\Delta n_{\mu}\right)=0,(\partial T / \partial t)=0$, and $\nabla T=0$. If we excite a single phonon at time $t_{0}$, its population relaxes back to equilibrium as $\Delta n_{\mu}(t)=\left(n_{\mu}\left(t_{0}\right)-\bar{n}_{\mu}\right) e^{-t / \tau_{\mu}^{\mathrm{SMA}}}$, i.e., with a characteristic time $\tau_{\mu}^{\mathrm{SMA}}$.

The thermal conductivity tensor $k^{i j}$ ( $i$ and $j$ are Cartesian indices) is defined as the ratio between a heat flux $Q^{i}$ and a static gradient of temperature $(\nabla T)^{j}$. Two simplifications apply in this case. First, a steady-state condition allows one to simplify the BTE by setting time derivatives to zero. Second, the spatial gradient can be simplified by taking $\nabla\left(\Delta n_{\mu}\right)=0$. This assumption, frequently adopted in the literature, holds for a homogeneous perturbation of a bulk crystal (as in our case): If we apply a thermal gradient to a crystal at temperature $T$, the response $\Delta n_{\mu}$ should not depend on the particular position $\boldsymbol{x}$ inside the sample. Although we will not consider it further here, we note that this assumption cannot be applied when studying systems that break translational invariance, involving, e.g., surfaces or pointlike heat sources. Under these conditions and the SMA, the resulting BTE can be solved analytically and, using the harmonic approximation for the heat flux $Q=(1 / \mathcal{V}) \sum_{\mu} \hbar \omega_{\mu} v_{\mu} \Delta n_{\mu}$ [34] and the definition $Q^{i}=-\sum_{j} k^{i j}(\nabla T)^{j}$, the thermal conductivity is given by

$$
\left(k^{i j}\right)^{\mathrm{SMA}}=\frac{1}{\mathcal{V}} \sum_{\mu} C_{\mu} v_{\mu}^{i}\left(\Lambda_{\mu}^{j}\right)^{\mathrm{SMA}},
$$


where $\left(\Lambda_{\mu}^{j}\right)^{\mathrm{SMA}}$ is the component of the phonon mean free path in direction $j$. This expression can be interpreted as the thermal conductivity of a gas of phonons, each carrying a specific heat $C_{\mu}=\left(1 / k_{B} T^{2}\right) \bar{n}_{\mu}\left(\bar{n}_{\mu}+1\right)\left(\hbar \omega_{\mu}\right)^{2}=$ $\left(\partial \bar{n}_{\mu} / \partial T\right) \hbar \omega_{\mu}$, traveling at velocity $v_{\mu}^{i}$ and with a mean free path $\left(\Lambda_{\mu}^{j}\right)^{\mathrm{SMA}}=v_{\mu}^{j} \tau_{\mu}^{\mathrm{SMA}}$ before being thermalized by scattering. Crucially, the definition of phonon lifetime or mean free path cannot be extended beyond the SMA since the offdiagonal terms of the scattering operator introduce couplings between phonons, and phonon thermalization stops being governed by an exponential relaxation.

\section{RELAXONS}

An exact definition of relaxation times has been formally derived by Hardy [22], as an auxiliary result in his study of second sound. To recall this definition, let us first note that the left side of the BTE in Eq. (2) has a drifting operator diagonal in $\mu$, whereas the right side has a scattering operator (determining scattering time scales) that is nondiagonal. To identify meaningful scattering times, we proceed with a change of basis that diagonalizes the scattering operator while allowing the drifting term to become nondiagonal. To make the symmetries more apparent within the BTE, we perform the transformations $[22,23,35,36]$

$$
\begin{gathered}
\tilde{\Omega}_{\mu \mu^{\prime}}=\Omega_{\mu \mu^{\prime}} \sqrt{\frac{\bar{n}_{\mu^{\prime}}\left(\bar{n}_{\mu^{\prime}}+1\right)}{\bar{n}_{\mu}\left(\bar{n}_{\mu}+1\right)},} \\
\Delta \tilde{n}_{\mu}=\left(\bar{n}_{\mu}\left(\bar{n}_{\mu}+1\right)\right)^{-\frac{1}{2}} \Delta n_{\mu} .
\end{gathered}
$$

These transformations are introduced to scale quantities appearing in the BTE in such a way that $\tilde{\Omega}_{\mu \mu^{\prime}}=\tilde{\Omega}_{\mu^{\prime} \mu}$ (the matrix $\Omega$ does not obey this symmetry; see Appendix A for a detailed explanation). We note that sometimes these transformations appear in the literature in the form of hyperbolic sines, by means of the identity $\sinh \left(\hbar \omega_{\mu} / 2 k_{B} T\right)=\left(1 / 2 \sqrt{\bar{n}_{\mu}\left(\bar{n}_{\mu}+1\right)}\right)$. Since $\tilde{\Omega}$ is a real symmetric matrix, it can be diagonalized, giving eigenvectors $\theta_{\mu}^{\alpha}$ and real eigenvalues $1 / \tau_{\alpha}$ such that

$$
\frac{1}{\mathcal{V}} \sum_{\mu^{\prime}} \tilde{\Omega}_{\mu \mu^{\prime}} \theta_{\mu^{\prime}}^{\alpha}=\frac{1}{\tau_{\alpha}} \theta_{\mu}^{\alpha},
$$

where $\alpha$ is the eigenvalue index. In passing, we define the scalar product $\left\langle\alpha \mid \alpha^{\prime}\right\rangle \equiv(1 / \mathcal{V}) \sum_{\mu} \theta_{\mu}^{\alpha} \theta_{\mu}^{\alpha^{\prime}}$ that allows us to define the orthonormalization condition for the eigenvectors $\left(\left\langle\alpha \mid \alpha^{\prime}\right\rangle=\delta_{\alpha \alpha^{\prime}}\right)$, which will be helpful in the next algebraic operations. It can be shown $[22,35]$ that $\tilde{\Omega}$ is positive-semidefinite, i.e., $\left(1 / \tau_{\alpha}\right) \geq 0 \forall \alpha$, and that its eigenvectors are either even or odd, i.e., $\theta_{\mu}^{\alpha}= \pm \theta_{-\mu}^{\alpha}$, where $-\mu=(-\boldsymbol{q}, s)$ [22]. Little else is known about the eigenvalue spectrum of Eq. (7), which therefore has to be characterized numerically. In contrast with Refs. [21,22], we remark that the Bose-Einstein distribution is not an eigenvector with zero eigenvalue: The scattering operator acts only on the deviation from equilibrium $\Delta n_{\mu}$; therefore, thermal equilibrium $\left(\Delta n_{\mu}=0\right)$ is a stationary solution $\left(\sum_{\mu^{\prime}} \Omega_{\mu \mu^{\prime}} \Delta n_{\mu}=0\right)$ because it is an algebraically trivial solution. However, the Bose-Einstein distribution allows the introduction of a vector of unitary length,

$$
\theta_{\mu}^{0}=\frac{\sqrt{\bar{n}_{\mu}\left(\bar{n}_{\mu}+1\right)} \hbar \omega_{\mu}}{\sqrt{k_{B} T^{2} C}},
$$

where $C=(1 / \mathcal{V}) \sum_{\mu} C_{\mu}$, describing the increase of temperature. This vector is constructed as the linear deviation from equilibrium of $\bar{n}_{\mu}(T+\delta T)$, transformed using Eq. (6) and normalized to 1 . Note that $\theta_{\mu}^{0}$ is not an eigenvector and that it does not have to be orthogonal to other eigenstates $\alpha$.

Any response $\Delta \tilde{n}_{\mu}$ can be written as a linear combination of the $\theta_{\mu}^{\alpha}$ eigenvectors [22]

$$
\Delta \tilde{n}_{\mu}(\boldsymbol{x}, t)=\sum_{\alpha} f_{\alpha}(\boldsymbol{x}, t) \theta_{\mu}^{\alpha},
$$

and the BTE can be written in this $\theta^{\alpha}$ basis (to this aim, substitute Eq. (9) in Eq. (2) and take the scalar product of the equation with a generic eigenvector $\alpha^{\prime}$ ), becoming

$$
\begin{aligned}
& \sqrt{\frac{C}{k_{B} T^{2}}}\left(\frac{\partial T(\boldsymbol{x}, t)}{\partial t}\langle 0 \mid \alpha\rangle+\nabla T(\boldsymbol{x}, t) \cdot \boldsymbol{V}_{\alpha}\right)+\frac{\partial f_{\alpha}(\boldsymbol{x}, t)}{\partial t} \\
& +\sum_{\alpha^{\prime}} \boldsymbol{V}_{\alpha \alpha^{\prime}} \cdot \nabla f_{\alpha^{\prime}}(\boldsymbol{x}, t)=-\frac{f_{\alpha}(\boldsymbol{x}, t)}{\tau_{\alpha}}
\end{aligned}
$$

where $\boldsymbol{V}_{\alpha \alpha^{\prime}}=(1 / \mathcal{V}) \sum_{\mu} \theta_{\mu}^{\alpha} v_{\mu} \theta_{\mu}^{\alpha^{\prime}} \equiv\left\langle\alpha|v| \alpha^{\prime}\right\rangle$ and $\boldsymbol{V}_{\alpha}=\boldsymbol{V}_{0 \alpha}=$ $\langle 0|v| \alpha\rangle . \boldsymbol{V}_{\alpha \alpha^{\prime}}$ derives from the action of the diffusion operator on the deviation from equilibrium, while $\boldsymbol{V}_{\alpha}$ derives from the action of the diffusion operator on the equilibrium distribution.

The physical picture encoded in Eq. (10) underlines one of the key statements of this work: By diagonalizing the scattering operator, the information about the characteristic relaxation time of the thermal excitations is now given by the eigenvalues $1 / \tau_{\alpha}$. The eigenvectors $\theta_{\mu}^{\alpha}$ for which this picture emerges represent collective excitations which we call relaxons. Each relaxon represents a distribution of phonon excitation numbers (a wave packet), describing how the phonon distribution is relaxing to equilibrium. The coefficients $f_{\alpha}$ are the relaxon occupation numbers, which are determined by the BTE in the out-of-equilibrium state and which at equilibrium are all 0 , so that the deviation from equilibrium $\Delta n_{\mu}$ vanishes. 


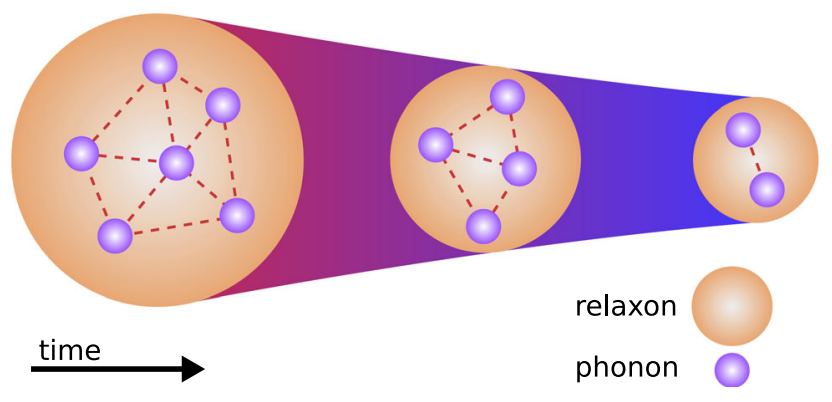

FIG. 1. Schematic illustration of the equilibration of lattice vibrations after a thermal excitation. Each relaxon consists of a linear combination of phonons, which interact through scattering events among themselves but are decoupled from phonons belonging to different relaxons. The relaxon decays exponentially to equilibrium, where it disappears at a rate determined by its relaxation time.

The name relaxon is easily justified by considering a system at thermal equilibrium so that $(\partial T / \partial t)=0$, $\nabla T=0$ and, in terms of relaxons, all states are empty everywhere; thus, $\nabla f_{\alpha}=0 \forall \alpha$ in Eq. (10). If we excite a single relaxon $\alpha$ at time $t_{0}$, its occupation will relax back to equilibrium as $f_{\alpha}(t)=f_{\alpha}\left(t_{0}\right) e^{-t / \tau_{\alpha}}$, therefore endowing $\tau_{\alpha}$ with the meaning of a relaxation time. Although the theory allows for zero eigenvalues, in our examples, we find only strictly positive relaxation times so that all relaxons decay to zero for $t \rightarrow \infty$. Using Eq. (9), one can show instead that phonon populations do not have welldefined relaxation times: Since each phonon population decays as a linear combination of relaxon processes $\Delta n_{\mu}(t)=\sum_{\alpha} f_{\alpha}\left(t_{0}\right) \theta_{\mu}^{\alpha} e^{-t / \tau_{\alpha}}$, the characteristic time for the decay depends on the initial conditions of the thermal excitation and can even display damped oscillations. Conversely, let us excite only one phonon mode $\mu$ at time $t_{0}$. This initial state can be decomposed as the sum of different relaxons, each evolving with a different relaxation time. Therefore, at a subsequent time $t$, one will also observe that new phonon modes $\mu^{\prime} \neq \mu$ have been excited out of thermal equilibrium. In Fig. 1, we graphically illustrate our interpretation: Each relaxon is a collective excitation of phonons, which interact through scattering events among themselves but are decoupled from phonons belonging to different relaxons; owing to their positive relaxation time, relaxons disappear at long times, allowing the system to reestablish equilibrium.

Velocities appear in Eq. (10) with a matrix $\boldsymbol{V}_{\alpha \alpha^{\prime}}$ coupling different relaxons since it is the phonon basis that diagonalizes the drifting operator; therefore, one cannot always identify a relaxon velocity. However, if we work in an infinite crystal at temperature $T$ and apply a temperature perturbation homogeneously to the entire crystal, the response of the system is constant throughout the space, and we can set $\nabla f_{\alpha}=0, \forall \alpha$. Therefore, the BTE simplifies to

$$
\frac{\partial f_{\alpha}(t)}{\partial t}+\sqrt{\frac{C}{k_{B} T^{2}}}\left(\frac{\partial T}{\partial t}\langle 0 \mid \alpha\rangle+\nabla T \cdot \boldsymbol{V}_{\alpha}\right)=-\frac{f_{\alpha}(t)}{\tau_{\alpha}}
$$

Both the drifting and the collision operator are now diagonal in $\alpha$, and $\boldsymbol{V}_{\alpha}$ identifies a well-defined relaxon velocity.

Let us simplify the problem further and consider a steady state. In this case, time derivatives are set to zero in Eq. (11), and one can, for small deviations from equilibrium, search for linear solutions of the form $f_{\alpha}=\sum_{i} f_{\alpha}^{i} \nabla_{i} T$, where $i$ is a Cartesian direction. The BTE reduces to

$$
\sqrt{\frac{C}{k_{B} T^{2}}} V_{\alpha}^{i}=-\frac{f_{\alpha}^{i}}{\tau_{\alpha}}
$$

whose solution for $f_{\alpha}^{i}$ is trivial. Using the relation between phonons and relaxon occupation numbers $\Delta n_{\mu}=\sqrt{\bar{n}_{\mu}\left(\bar{n}_{\mu}+1\right)}\left(\sum_{i \alpha} \nabla_{i} T f_{\alpha}^{i} \theta_{\mu}^{\alpha}\right)$, we obtain the thermal conductivity

$$
\begin{aligned}
k^{i j} & =\frac{-1}{\mathcal{V} \nabla_{i} T} \sum_{\mu} \hbar \omega_{\mu} v_{\mu}^{j} \Delta n_{\mu}=-\sum_{\alpha} f_{\alpha}^{i} \sqrt{k_{B} T^{2} C} V_{\alpha}^{j} \\
& =\sum_{\alpha} C V_{\alpha}^{i} V_{\alpha}^{j} \tau_{\alpha}=\sum_{\alpha} C V_{\alpha}^{i} \Lambda_{\alpha}^{j},
\end{aligned}
$$

where we introduced the relaxon mean free path $\Lambda_{\alpha}\left(\Lambda_{\alpha}^{j}\right.$ is the component of $\Lambda_{\alpha}$ in direction $j$ ). Therefore, the exact thermal conductivity in Eq. (13) is expressed in the framework of the kinetic theory of gases, and thermal transport can be thought of as a flux of relaxons, each carrying a specific heat $C$, traveling at velocity $V_{\alpha}$ for an average distance of $\Lambda_{\alpha}$ before thermalization occurs.

At variance with the phonon picture, where each phonon participates in thermal conductivity with a mode specific heat $C_{\mu}$, all relaxons contribute with the same specific heat of the crystal $C$. Mathematically, the phonon-mode specific heat is moved in the vector $\theta_{\mu}^{0}$ (note that $\left(\theta_{\mu}^{0}\right)^{2}=\left(C_{\mu} / C\right)$ ) and thus is included in the relaxon velocity $V_{\alpha}=\langle 0|v| \alpha\rangle$. To physically interpret this difference, we recall that, from a thermodynamic point of view, the quantity $C \delta T$ is the energy needed by the system to change the temperature by $\delta T$. To observe such a temperature change, all phonon modes must simultaneously change their occupation number according to the collective excitation $\theta_{\mu}^{0}$. The quantity $C_{\mu} \delta T$ is the decomposition of such energy change in terms of each phonon mode. However, as explained before, one cannot excite a single phonon mode and bring it to a higher temperature without affecting the rest of the phonon ensemble: Phonon scattering would redistribute the energy excess of such a mode to the rest of the system. Only a collective excitation of phonons $\left(\theta_{\mu}^{0}\right)$ leads to a temperature change, and the total energy cost for increasing temperature is necessarily associated with $C$; thus, from a thermodynamic point of view, one could state that the 
mode specific heat $C_{\mu}$ does not have a well-defined meaning.

It is worth pointing out the role played by the parity of relaxons. The quantity $\boldsymbol{V}_{\alpha}=\langle 0|v| \alpha\rangle$ involves the odd function $v_{\mu}\left(-v_{\mu}=v_{-\mu}\right)$ and the even function $\theta_{\mu}^{0}$ (owing to $\omega_{\mu}=\omega_{-\mu}$ ). Therefore, relaxon velocities $\boldsymbol{V}_{\alpha}$ are different from zero only for odd relaxons $\alpha$. Consequently, Eq. (12) also implies that only odd relaxons are excited in the steady-state condition and thus contribute to heat flux, while even relaxons have zero occupation number. The role of parity is reversed for determining the energy of the system since the change from equilibrium energy $\Delta E$ is

$$
\begin{aligned}
\Delta E & =\frac{1}{\mathcal{V}} \sum_{\mu} \hbar \omega_{\mu} \Delta n_{\mu}=\frac{1}{\mathcal{V}} \sum_{\mu} \hbar \omega_{\mu} \sqrt{\bar{n}_{\mu}\left(\bar{n}_{\mu}+1\right)} \Delta \tilde{n}_{\mu} \\
& =\frac{1}{\mathcal{V}} \sum_{\mu} \theta_{\mu}^{0} \sqrt{k_{B} T^{2} C} \sum_{\alpha} f_{\alpha} \theta_{\mu}^{\alpha} \\
& =\sqrt{k_{B} T^{2} C} \sum_{\alpha} f_{\alpha}\langle 0 \mid \alpha\rangle .
\end{aligned}
$$

In this case, even relaxons have a nonzero coefficient $\langle 0 \mid \alpha\rangle$ and contribute to an energy change, but for odd relaxons, $\langle 0 \mid \alpha\rangle=0$ and thus do not change energy. We can thus deduce that at the steady state defined by Eq. (11), where only odd relaxons are excited, the energy of the system is conserved.

\section{GRAPHENE}

As a first numerical example supporting these conclusions, we study relaxons in graphene, the material with the highest known thermal conductivity [37], and contrast the phonon and the relaxon pictures at $300 \mathrm{~K}$. Because of its symmetry, graphene's $k^{i j}$ tensor is diagonal and, since $k^{x x}=k^{y y}$, it has only one independent component (verified also numerically); therefore, in the following we will drop Cartesian indices and compute quantities numerically along the zig-zag direction. To proceed, we calculate harmonic and anharmonic force constants using density-functional perturbation theory [38-44] as implemented in the Quantum-ESPRESSO distribution [45] and construct the scattering matrix using three-phonon and isotope-phonon interactions. The diagonalization of Eq. (7) provides all the relaxon eigenvectors $\theta_{\mu}^{\alpha}$; each of them represents, at fixed relaxon index $\alpha$, a difference in phonon populations with respect to thermal equilibrium [provided that it is backtransformed using Eq. (6)]. Notably, only a few $\theta_{\mu}^{\alpha}$ have large relaxation times; for example, the longest-lived relaxon $(\alpha=1)$ is plotted in Fig. 2 as a function of the phonon index $\mu=(\boldsymbol{q}, s)$. The first three $(s=1,2,3)$ branches are shown, corresponding to the out-of-plane, transverse, or longitudinal acoustic phonons (ZA, TA. and LA, respectively). This particular relaxon induces a population difference for the ZA branch mainly located

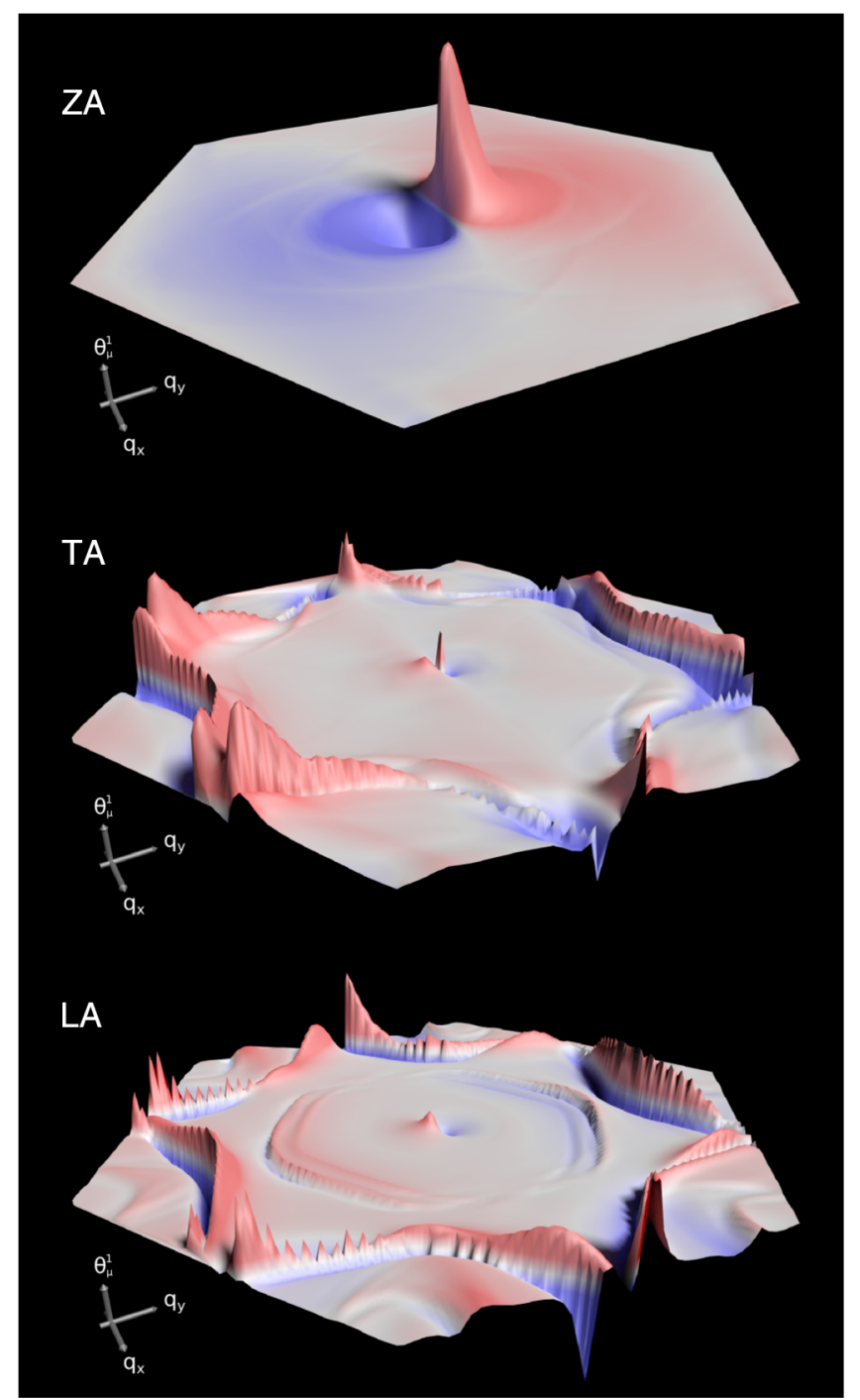

FIG. 2. Representation of the relaxon $\theta_{\mu}^{\alpha}$ with the longest relaxation time $(\alpha=1)$ in graphene at room temperature as a function of the phonon index $\mu=(\boldsymbol{q}, s)$, where we choose $s$ to be the out-of-plane, transverse, or longitudinal acoustic mode (ZA, TA, and LA, respectively). We recall that the relaxon is a difference in phonon populations with respect to thermal equilibrium: Overpopulated modes are shown in red; depopulated ones are in blue. The fine structure of the ridges is a numerical artifact due to discrete Brillouin-zone sampling.

close to the Brillouin-zone center, whereas TA and LA modes are altered throughout the Brillouin zone. The variations of optical modes ( $s=4,5,6$ not shown) are an order of magnitude smaller. The complex landscape drawn by these phonon distributions reflects the fact that out-of-equilibrium lattice properties cannot be described in terms of single phonon properties, as the action of scattering tightly couples phonons of any wave vector and branch.

We analyze the entire phonon and relaxon spectrum in Fig. 3, where the contributions to the SMA or the exact thermal conductivities are plotted as a function of the relaxon or phonon relaxation times. The thermal 


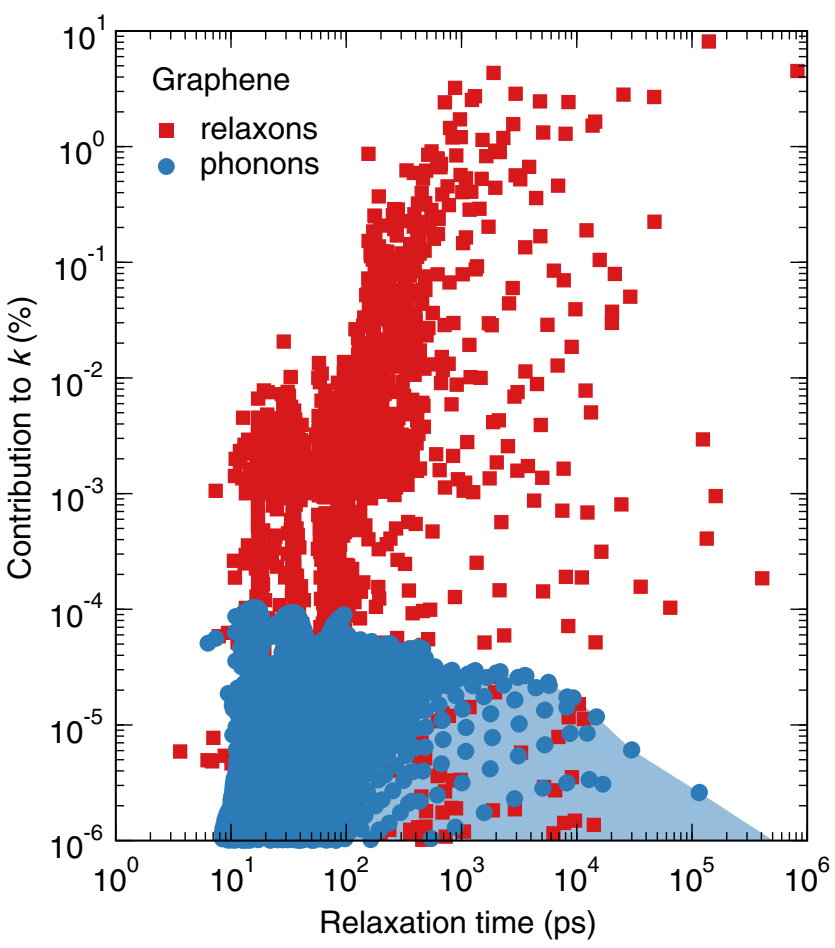

FIG. 3. Relaxation times and their contribution to the thermal conductivity of graphene at room temperature, considering relaxons or phonons as heat carriers. Relaxons tend to be longer lived than single phonon excitations, with large contributions to thermal conductivity coming from excitations with relaxation time larger than $10^{3} \mathrm{ps}$, whereas phonons have lifetimes mainly in the range $10-100 \mathrm{ps}$. The shaded area is a guide to the eyes to stress that phonons form a continuous spectrum, while relaxons are discretized; thermal conductivity can be accurately described using a small number of relaxons.

conductivities computed in the two pictures differ significantly in graphene (in this work, we compute $3894 \mathrm{~W} / \mathrm{mK}$ with the exact BTE against $495 \mathrm{~W} / \mathrm{mK}$ with the SMA); hence, to have more comparable quantities, we plot the percentage contribution to thermal conductivity. We first note that the spectrum of phonon lifetimes (and phonon velocities and mean free paths) is continuous, with even a divergence $\tau_{\mu} \rightarrow \infty$ for acoustic ZA phonons at the $\Gamma$ point in isotopically pure samples [46]. This divergence cannot be accurately described with a finite mesh of points sampling the Brillouin zone (in our case, a full mesh of $128 \times 128$ points), resulting in a sparse tail of long-lived phonons on the right side of Fig. 3, whose contribution to $k^{\text {SMA }}$ is negligible [46]. Instead, relaxation times for relaxons are discrete and sparse, in particular, in the region of large values, so that only a small number of relaxons is sufficient to describe thermal transport with high accuracy. This observation is robust with respect to Brillouin-zone sampling: With an improvement in the integration mesh, new phonon modes appear in the long-lifetime region; instead, the longest relaxon relaxation times convergefrom above - to the discretized values shown in the figure.

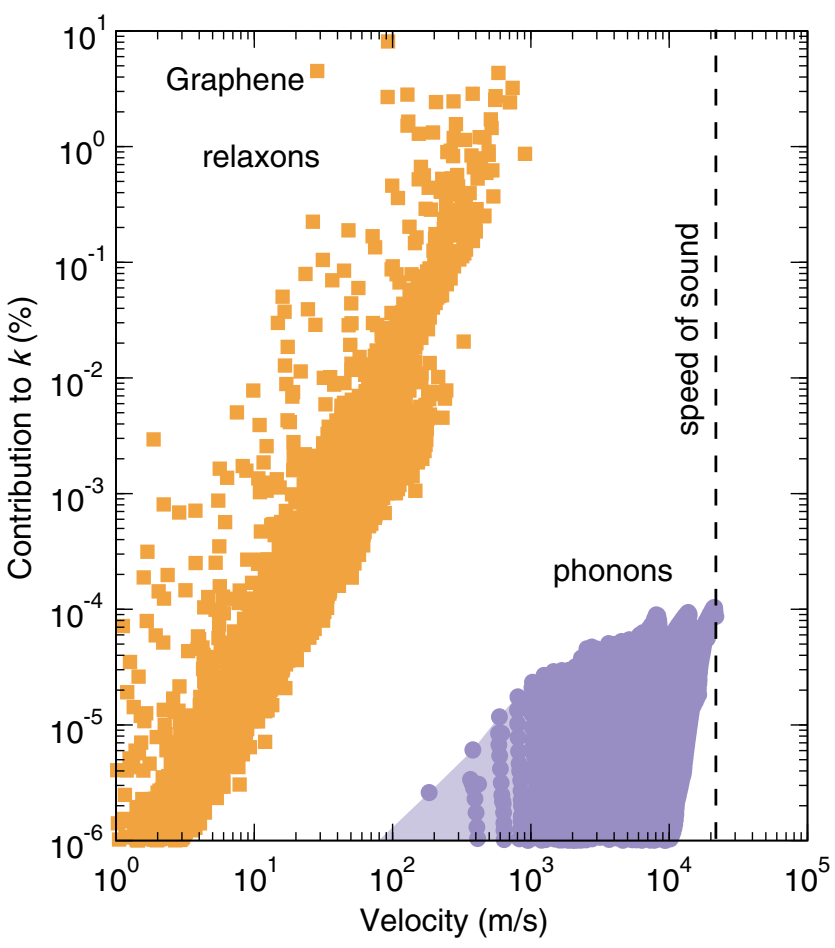

FIG. 4. Comparison of relaxon and phonon velocities and their contributions to the room-temperature thermal conductivity of graphene. When approximating phonons as heat carriers, the velocity scale of thermal transport is set by the speed of sound (about $20 \mathrm{~km} / \mathrm{s}$ for longitudinal acoustic phonons in graphene). Instead, relaxon velocities are at least an order of magnitude smaller, illustrating how much the phonon scattering slows down the heat flux.

On average, relaxon relaxation times are larger by at least 2 orders of magnitude with respect to phonon lifetimes. The large difference between the time scales of phonons and relaxons appears because a single phonon scattering cannot thermalize the system [18], as implied by the SMA. Therefore, while phonons scatter at time scales of about 10-100 ps, heat flux is dissipated by relaxons within nanosecond and microsecond time scales.

Before analyzing velocities, we note that the sign of $V_{\alpha}$ is arbitrary since both $\theta_{\mu}^{\alpha}$ and $-\theta_{\mu}^{\alpha}$ are relaxon eigenvectors. As a convention, we select the sign of odd eigenvectors such that $V_{\alpha}$ is non-negative (as is $\Lambda_{\alpha}$ ), noting that in any case, the contribution to $k$ would be positive (as $V_{\alpha}^{2}$ ). Phonon velocities can also assume both signs: In the figure, we plot their absolute values. Figure 4 reveals that the velocities of relaxons are much smaller than those of phonons: While the scale of phonon velocities is set by the speed of sound (the group velocity of the longitudinal acoustic phonon is about $20 \mathrm{~km} / \mathrm{s}$ ), relaxons are slower by 2 orders of magnitude, indicating that heat is transferred through the material at $0.1-1 \mathrm{~km} / \mathrm{s}$.

Finally, we show the relaxon mean free paths in Fig. 5 (projected along the transport direction). As other 


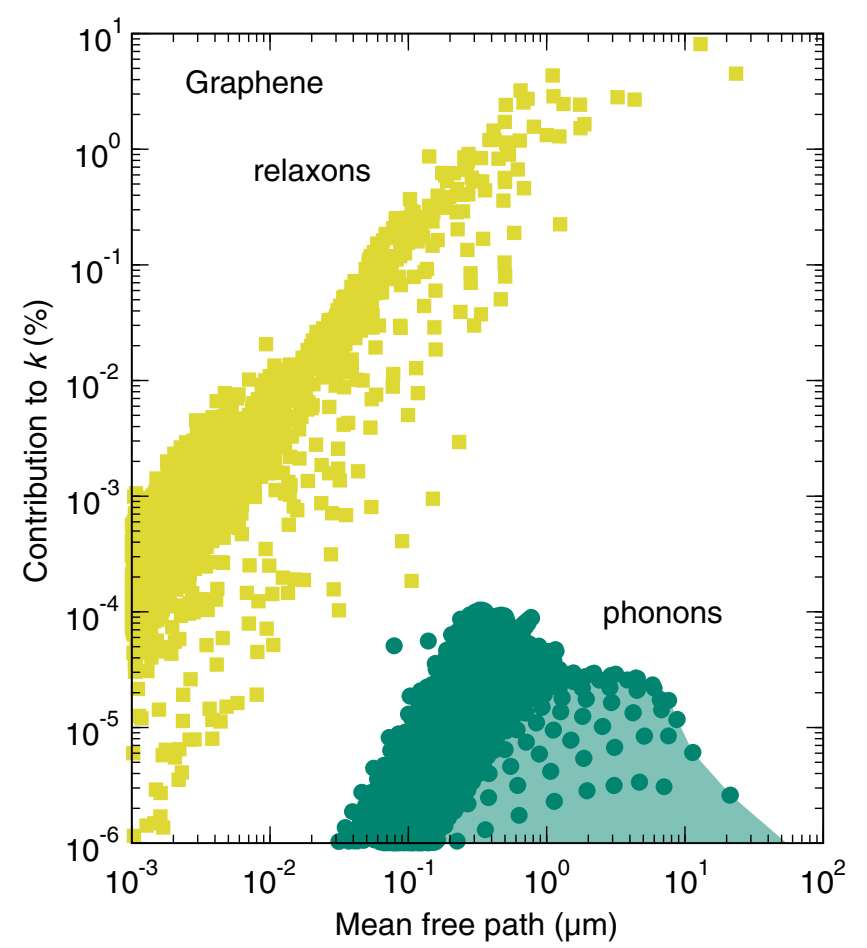

FIG. 5. Analysis of phonon and relaxon mean free paths and their contributions to the thermal conductivity of graphene at room temperature. The spectrum of phonon mean free paths is peaked in the submicron region, whereas relaxon mean free paths are skewed to values larger than $1 \mu \mathrm{m}$. The two largest relaxon mean free paths, illustrating the maximum distance that heat flux can propagate inside the material before decaying, lie between 10 and $100 \mu \mathrm{m}$.

first-principles studies reported [8], phonon mean free paths for graphene are distributed in the $0.1-1 \mu \mathrm{m}$ region [29]; this is confirmed here. For relaxons, most contributions to thermal conductivity come with mean free paths above $0.1 \mu \mathrm{m}$, the longest and most important contributions having mean free paths up to tens of $\mu \mathrm{m}$. The contribution to $k$ is roughly monotonic with the mean free path, and the large increase in $\tau_{\alpha}$ is partly compensated by the decreased $V_{\alpha}$. The saturation of relaxons' mean free paths at tens of $\mu \mathrm{m}$ appears to be in contrast with recent estimates for saturation lengths of $100 \mu \mathrm{m}$ [29] or longer [47]; we will comment on this discrepancy after discussing the next example.

\section{SILICON}

As a further test for relaxons, let us now turn our attention to silicon and examine its thermal transport properties at room temperature. The thermal conductivity tensor in silicon is diagonal, and the three Cartesian directions are equivalent; we therefore only consider transport properties along the (100) direction. At variance with graphene, here the SMA introduces an appealingly small error: In our calculations, we find $138 \mathrm{~W} / \mathrm{mK}$ instead of $141 \mathrm{~W} / \mathrm{mK}$ for the exact solution; these estimates are in

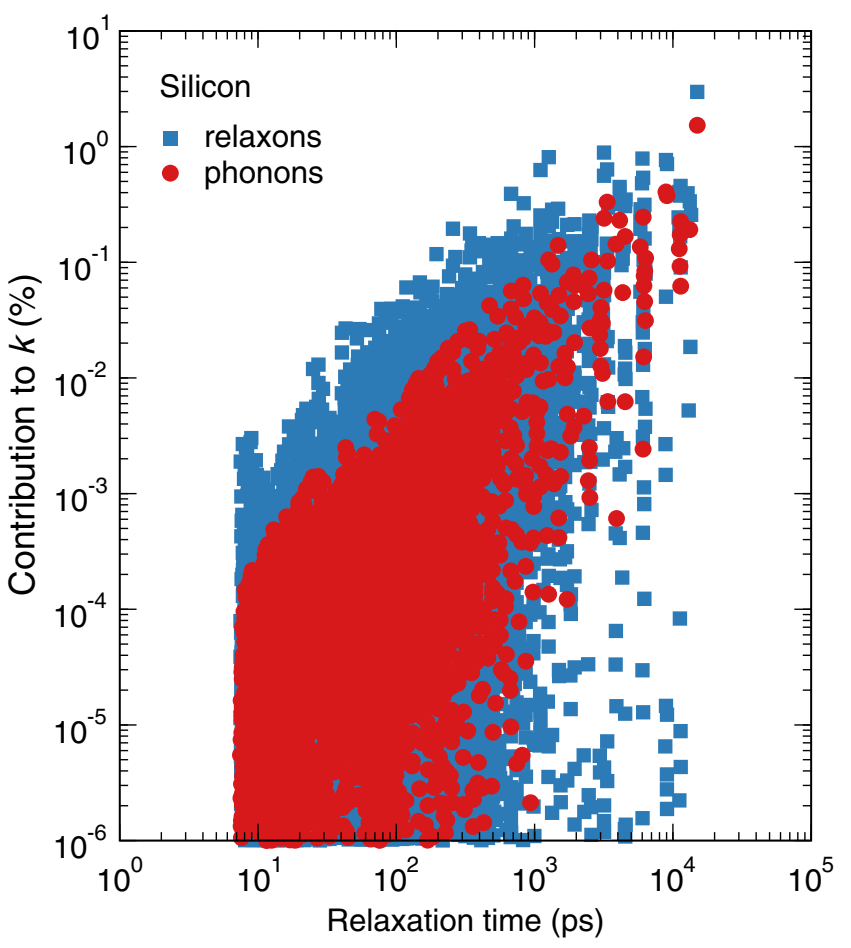

FIG. 6. Same as in Fig. 3, this time for silicon at room temperature, the spectrum of relaxation times, and their contribution to thermal conductivity, considering relaxons or phonons as heat carriers. In this material, the relaxation times estimated with the SMA are relatively close to the exact relaxation times of the system, with contributions ranging from a few picoseconds up to approximately $10^{4} \mathrm{ps}$.

line with previous first-principles studies $[5,6,8]$. The small difference between the two pictures is somehow replicated in their relaxation times, as reported in Fig. 6. The time scales covered by phonons and relaxons have approximately the same range of values, except for one relaxon, not shown in the graph, that has relaxation time of $2 \times 10^{5} \mathrm{ps}$ but a negligible contribution to thermal conductivity $\left(10^{-9} \%\right)$. However, one can note that the two distributions of values do not perfectly overlap: Even if the scattering matrix is diagonally dominant, there are small nonzero outof-diagonal matrix elements that introduce deviations from the SMA.

It is enlightening to analyze the different velocity scales set by the two pictures, depicted in Fig. 7. Once again, most of the contributions to SMA thermal transport come from phonons with velocities close to the speed of sound, which in silicon is approximately $8 \mathrm{~km} / \mathrm{s}$. However, relaxon velocities are 2 orders of magnitude smaller than this limiting value, reaching merely $60 \mathrm{~m} / \mathrm{s}$. Despite the fact that the instantaneous velocity of a lattice vibration is determined by the phonon dispersion, the velocity at which the heat flux propagates can be much different: The scattering between phonons slows it down.

The mean free paths for relaxons and phonons in silicon are compared in Fig. 8. The vast difference originating from 


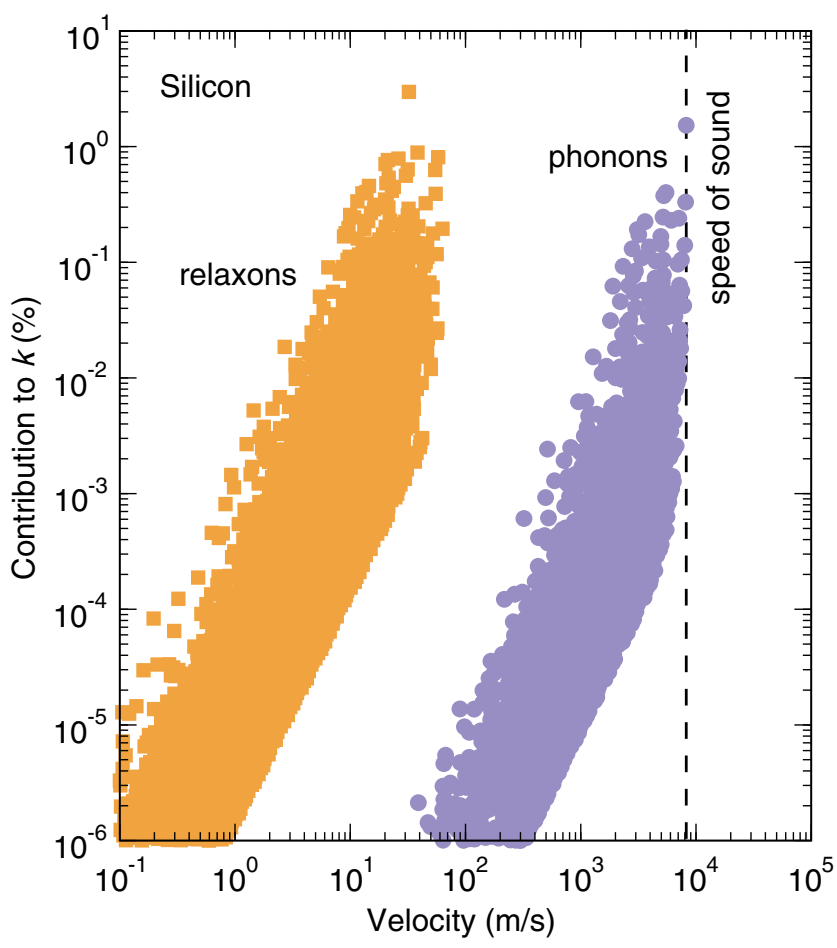

FIG. 7. Same as in Fig. 4 but for silicon, the comparison of relaxons and phonon velocities and their contributions to roomtemperature thermal conductivity. Similar to the case of graphene, the velocity of the SMA description is set by the velocity of phonons, with long-wavelength acoustic modes (those with the highest velocities) giving the largest contributions. Relaxon velocities are instead smaller by about 2 orders of magnitude, indicating that the heat flux is slowed down by the action of phonon scattering.

the velocities is carried over, so while mean free paths of phonons extend up to $100 \mu \mathrm{m}$, in agreement with other first-principles studies $[8,48,49]$, relaxons travel for a distance that is 2 orders of magnitude smaller than phonons. Therefore, it seems puzzling that the two pictures give such large differences in the estimate of velocities and mean free paths, despite the fact that thermal conductivities are essentially identical. To explain this discrepancy, we compare Eqs. (4) and (13) for thermal conductivity and recall that specific heat is constant for relaxons and mode specific for phonons. Also, the SMA conductivity can be written in a form with constant specific heat for each phonon, provided that we rescale velocities as $v_{\mu} \rightarrow$ $\sqrt{\left(C_{\mu} / \mathcal{V} C\right)} v_{\mu}$ (consequently, the mean free path is scaled as $\left.\Lambda_{\mu}^{\mathrm{SMA}}=v_{\mu} \tau_{\mu}^{\mathrm{SMA}} \rightarrow \sqrt{\left(C_{\mu} / \mathcal{V} C\right)} \Lambda_{\mu}^{\mathrm{SMA}}\right)$. After this transformation, velocities and mean free paths of phonons and relaxons in silicon are again within the same order of magnitude, although residual discrepancies still persist (see Appendix B for the spectra after rescaling). Therefore, the differences observed in silicon between the two pictures arise mainly from the different interpretation of specific heat.

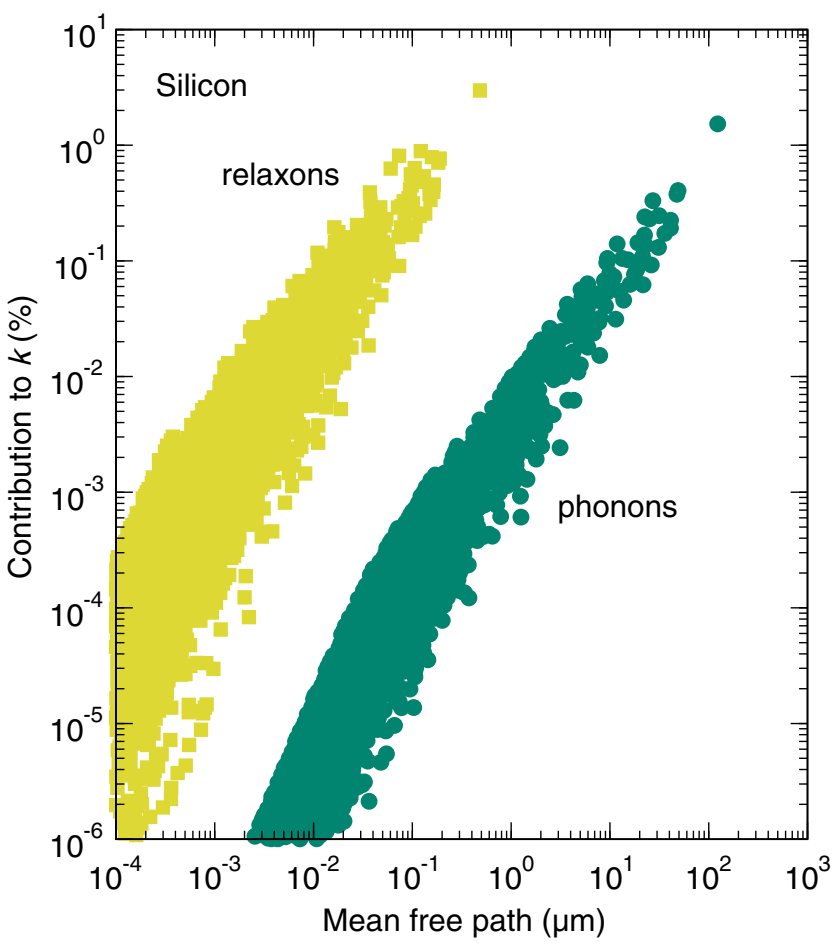

FIG. 8. Analysis of phonon and relaxon mean free paths and their contributions to the thermal conductivity of silicon at room temperature. The difference between velocities is carried over to this spectrum, so relaxon mean free paths are shifted to smaller values with respect to phonons. While our estimates of mean free paths for phonons extend up to hundreds of micrometers, relaxon mean free paths barely reach the micrometer scale.

We note that other experimental and theoretical efforts have estimated heat mean free paths in silicon [8,48-52], obtaining values that are comparable to those of the phonon mean free paths and different from the relaxon mean free paths presented here. It is important to stress, though, that at least one of these two assumptions was used: first, that results can be interpreted considering phonons as the heat carriers, and second, that surface or grain boundary scattering can be exploited as a tool to estimate heat mean free paths. In the present work, we already discussed at length the limitations of the former assumption; as for the latter one, we cannot compare our results with studies that rely on surface scattering since the data presented here pertain to a homogeneous bulk crystal. It is nevertheless possible to drop the bulk condition and solve the BTE in the presence of surfaces, reconciling the different pictures; such a discussion is out of the scope of the present work and will be presented in an upcoming study.

\section{FURTHER PROPERTIES}

A widely held assumption that is also violated by the exact BTE is the Matthiessen rule, which states that the total thermal resistivity (i.e., $1 / k$ ) is the sum of the 

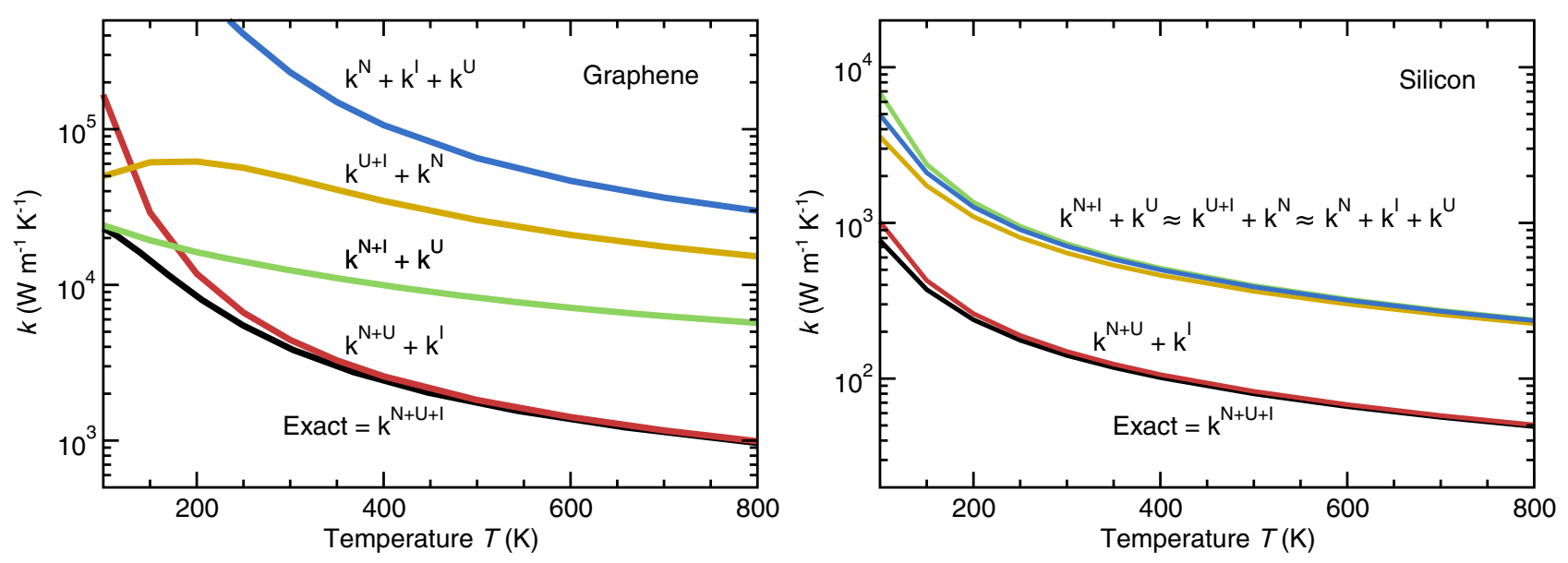

FIG. 9. Study of the failure of the Matthiessen rule in graphene and silicon. The total thermal conductivity (black line) is compared to conductivities computed through various Matthiessen sums, where the total sum is given by the sum of the reciprocals (by $k^{N}+k^{U}+k^{I}$, we mean $\left.k^{-1}=\left(k^{N}\right)^{-1}+\left(k^{U}\right)^{-1}+\left(k^{I}\right)^{-1}\right)$. Because of the correlation between scattering events, there is no decomposition for which the Matthiessen rule is obeyed at all temperatures. The only curve that approaches the exact thermal conductivity is the decomposition $\left(k^{N+U}+k^{I}\right)$, just when the effect of isotopic scattering is negligible.

resistivities of each independent scattering mechanism; however, the Matthiessen rule is an approximation [18] relying on the possibility of exactly decoupling scattering mechanisms. To numerically probe this violation, we computed the resistivities of normal, Umklapp, and isotopic processes, or any combination of these, and combined them according to the Matthiessen rule. In Fig. 9, we show that, regardless of any particular decomposition, the conductivity obtained by imposing the Matthiessen sum deviates significantly from the exact conductivity. The only case in which a decomposition reproduces the exact result is when the effect of a separated mechanism is negligible; for the case shown in the figure, one can separately sum the resistance due to isotopes only at high temperatures, when it is small. Finally, one can prove that the total thermal conductivity is always smaller than or equal to the Matthiessen sum (see Ref. [18] or Appendix C); this result is also verified in our calculations. Moreover, at variance with the case of phonon lifetimes, it is not possible to identify the contributions to the relaxation times coming from each scattering mechanism: in fact, when a scattering matrix is not diagonal, the eigenvalues of the sum of two matrices are not the sum of the eigenvalues of two matrices.

As an added benefit, the direct diagonalization of the scattering matrix brings clear insight into the numerical stability of current methods used to solve the BTE. In particular, we show in Appendix D that the iterative method $[4,16,17]$, often used to study 2D materials, is numerically unstable for graphene at room temperature, because of the dominant contribution of the out-of-diagonal terms in the scattering matrix (this is exactly the case when the relaxon picture differs significantly from the phonon picture).

\section{CONCLUSIONS}

In summary, we have shown that by choosing the eigenvectors of the scattering matrix as a basis, the linear BTE can be greatly simplified. These eigenvectors are collective excitations of phonon populations, termed relaxons, that have well-defined relaxation times and, in the homogeneous steady-state case, also proper velocities and mean free paths. Thermal transport can be described as a kinetic theory of the relaxon gas, thus with time, velocity and length scales determined by relaxons. For clarity, we report in Table I a summary of relaxon characteristics and how they compare with phonons. This theory is applied here first to graphene at room temperature where, as is typical of $2 \mathrm{D}$ materials or of 3D solids at low temperatures, the failure of the SMA and of its picture of phonons as heat carriers becomes dramatic; and to silicon at room temperature, where, although the SMA yields reasonable thermal conductivities, the theory brings new insight in the microscopic interpretation of heat flux and its typical velocities. Finally, we have shown that the Matthiessen rule is violated in the exact BTE, with significant consequences for all systems in which the SMA does not hold. As a final remark, the concept of relaxons has been applied in this work in the context of phonons; however, similar arguments hold for the electron BTE or other semiclassical transport models.

\section{METHODS}

\section{A. First-principles simulations}

Density-functional theory calculations have been performed with the Quantum-ESPRESSO distribution [45], 
TABLE I. A comparison of the main properties of phonons and relaxons.

\begin{tabular}{|c|c|c|}
\hline & Phonon & Relaxon \\
\hline Definition & Eigenstate of harmonic Hamiltonian & Eigenstate of collision matrix \\
\hline Physical meaning & $\begin{array}{c}\text { Collective excitation of atomic displacements } \\
\text { Quantum of vibrational energy }\end{array}$ & $\begin{array}{c}\text { Collective excitation of phonon populations } \\
\text { Elementary carrier of heat }\end{array}$ \\
\hline Exact quantities & $\begin{array}{c}\text { Lifetime, velocity and mean free } \\
\text { path of the vibration } \\
\text { Quasiparticle (energy, wave vector, dispersion relations) }\end{array}$ & $\begin{array}{c}\text { Relaxation time, velocity and mean free } \\
\text { path of the heat carrier } \\
\text { No dispersion relations }\end{array}$ \\
\hline Thermal conductivity & Only obtained as solution of the BTE & Obtained as a kinetic theory of the relaxon gas \\
\hline
\end{tabular}

using the local-density approximation and norm-conserving pseudopotentials from the PSLibrary [53]; for graphene, a plane-wave cutoff of $90 \mathrm{Ry}$ and a Methfessel-Paxton smearing of 0.02 Ry have been used, and for silicon, a plane-wave cutoff of 100 Ry has been used. Graphene is simulated with a slab geometry, using an optimized lattice parameter $a=4.607$ Bohr and a cell height $c=3 a$; for silicon, we find an optimized lattice parameter of 10.18 Bohr. The Brillouin zone is integrated with a Gammacentered Monkhorst-Pack mesh of $24 \times 24 \times 1$ points for graphene and $12 \times 12 \times 12$ for silicon. Second- and thirdorder force constants are computed on meshes of $16 \times 16 \times 1$ and $4 \times 4 \times 1$ points, respectively, for graphene and $8 \times 8 \times 8$ and $4 \times 4 \times 4$ for silicon, and they are later Fourier interpolated on finer meshes.

\section{B. Thermal conductivity simulations}

The scattering matrix $\tilde{\Omega}$ includes three-phonon interactions and harmonic isotopic scattering [6,7] at natural abundances [54] $\left(98.93 \%{ }^{12} \mathrm{C}, 1.07 \%{ }^{14} \mathrm{C}\right.$ for carbon, and $92.22 \%{ }^{28} \mathrm{Si}, 4.67 \%{ }^{29} \mathrm{Si}, 3.09 \%{ }^{30} \mathrm{Si}$ for silicon). For graphene, the scattering matrix is constructed using the same computational parameters of Ref. [29] (a Gaussian smearing of $10 \mathrm{~cm}^{-1}$ and a mesh of $128 \times 128 \times 1$ points for integrating the Brillouin zone), resulting in a matrix of order 98304, while for silicon, we use a Gaussian smearing of $7 \mathrm{~cm}^{-1}$ and a mesh of $30 \times 30 \times 30$, yielding a matrix of order 162000 . Here, $\tilde{\Omega}$ is diagonalized exactly using the routine PDSYEV of the Scalapack library [55]. The simulation cell of graphene is renormalized using the interlayer distance of bulk graphite $(c / a=1.367)$, in order to have a thermal conductivity comparable with the 3D counterpart. We verified the correctness of the software implementation ensuring that the thermal conductivity estimated with the diagonalization solver coincides with that computed with the variational method of Ref. [7] up to at least four significant digits. It is worth mentioning that these calculations are not prohibitively expensive and could be extended to other systems. The present software implementation computes $\tilde{\Omega}$, diagonalizes it, and computes the conductivity of graphene in about 5 hours using 256 CPUs on the Piz Daint supercomputer of the Swiss National Supercomputer Center (CSCS), for a total of 1300 CPU hours (1000 of which are spent in the diagonalization). For silicon, the calculation was completed in 8 hours on $576 \mathrm{CPUs}$, for a total of $4600 \mathrm{CPU}$ hours. Calculations have been managed using the AiiDA materials' informatics platform [56].

\section{ACKNOWLEDGMENTS}

We gratefully acknowledge F. Mauri and G. Fugallo for useful discussions, the Swiss National Science Foundation under Project ID 200021_143636 and the National Centre of Competence in Research MARVEL, the Max PlanckEPFL Center for Molecular Nanoscience and Technology, and the Swiss National Supercomputing Center CSCS under Project ID s580.

\section{APPENDIX A: SCATTERING RATES}

In this appendix, we report the expressions for building the scattering matrix using three-phonon and isotope scattering events, which are discussed in detail in Ref. [7]. To make a connection with other studies, we note that most solve the BTE using a phonon deviation from equilibrium of the form $n_{\mu}=\bar{n}_{\mu}+\bar{n}_{\mu}\left(\bar{n}_{\mu}+1\right) F_{\mu}$. Since the action of the collision operator must not change, we have the relation

$$
\sum_{\mu^{\prime}} A_{\mu \mu^{\prime}} F_{\mu^{\prime}}=\sum_{\mu^{\prime}} \Omega_{\mu \mu^{\prime}} \Delta n_{\mu^{\prime}}
$$

where $A$ is the scattering matrix when it acts on $F$, related with the scattering matrices used in our work by

$$
\begin{aligned}
& \Omega_{\mu \mu^{\prime}}=A_{\mu \mu^{\prime}} \frac{1}{\bar{n}_{\mu^{\prime}}\left(\bar{n}_{\mu^{\prime}}+1\right)}, \\
& \tilde{\Omega}_{\mu \mu^{\prime}}=\frac{1}{\sqrt{\bar{n}_{\mu}\left(\bar{n}_{\mu}+1\right)}} A_{\mu \mu^{\prime}} \frac{1}{\sqrt{\bar{n}_{\mu^{\prime}}\left(\bar{n}_{\mu^{\prime}}+1\right)}} .
\end{aligned}
$$

The scattering rate for a phonon coalescence event is

$$
\begin{aligned}
P_{\mu \mu^{\prime}}^{\mu^{\prime \prime}}= & \frac{2 \pi}{N \hbar^{2}} \sum_{\boldsymbol{G}}\left|V^{(3)}\left(\mu, \mu^{\prime},-\mu^{\prime \prime}\right)\right|^{2} \bar{n}_{\mu} \bar{n}_{\mu^{\prime}}\left(\bar{n}_{\mu^{\prime \prime}}+1\right) \delta_{\boldsymbol{q}+\boldsymbol{q}^{\prime}-q^{\prime \prime}, \boldsymbol{G}} \\
& \times \delta\left(\hbar \omega_{\mu}+\hbar \omega_{\mu^{\prime}}-\hbar \omega_{\mu^{\prime \prime}}\right),
\end{aligned}
$$


where $N$ is the number of $\boldsymbol{q}$ points, $\boldsymbol{G}$ is a reciprocal lattice vector, and $V^{(3)}$ is the third derivative of the unit-cell energy $\mathcal{E}^{\text {cell }}$ with respect to atomic displacements,

$$
V^{(3)}\left(\mu, \mu^{\prime}, \mu^{\prime \prime}\right)=\frac{\partial^{3} \mathcal{E}^{\mathrm{cell}}}{\partial X_{\mu} \partial X_{\mu^{\prime}} \partial X_{\mu^{\prime \prime}}},
$$

with

$$
X_{\boldsymbol{q} s}=\frac{1}{N} \sum_{l b \alpha} \sqrt{\frac{2 M_{b} \omega_{\boldsymbol{q} s}}{\hbar}} z_{\boldsymbol{q} s}^{*, b \alpha} u_{b \alpha}\left(\boldsymbol{R}_{l}\right) e^{-i \boldsymbol{q} \cdot \boldsymbol{R}_{l}},
$$

where $b$ is an index running on the basis of atoms in the unit cell, $\boldsymbol{R}_{l}$ is a Bravais lattice vector identifying the $l$ th unit cell inside the crystal, $\alpha$ is a Cartesian index, $M_{b}$ is the mass of atom $b, z$ is the phonon polarization vector, and $u$ is the vector of atomic displacements. The scattering rate for a phonon-isotope scattering event is

$$
\begin{aligned}
P_{\mu \mu^{\prime}}^{\text {isot }} & =\frac{\pi}{2 N} \omega_{\mu} \omega_{\mu^{\prime}}\left[\bar{n}_{\mu} \bar{n}_{\mu^{\prime}}+\frac{\bar{n}_{\mu}+\bar{n}_{\mu^{\prime}}}{2}\right] \\
& \times \sum_{b} g_{b}\left|\sum_{\alpha} z_{\mu}^{*, b \alpha} z_{\mu^{\prime}}^{b \alpha}\right|^{2} \delta\left(\omega_{\mu}-\omega_{\mu^{\prime}}\right),
\end{aligned}
$$

where $g_{b}=\left(\left(M_{b}-\left\langle M_{b}\right\rangle\right)^{2} /\left\langle M_{b}\right\rangle^{2}\right)$.

Combining these scattering rates, the scattering matrix $A$ is

$$
\begin{aligned}
A_{\mu \mu^{\prime}}= & {\left[\sum_{\mu^{\prime \prime}, \mu^{\prime \prime \prime}}\left(P_{\mu \mu^{\prime \prime \prime}}^{\mu^{\prime \prime}}+\frac{1}{2} P_{\mu^{\prime \prime} \mu^{\prime \prime \prime}}^{\mu}\right)+\sum_{\mu^{\prime}} P_{\mu \mu^{\prime}}^{\mathrm{isot}}\right] \delta_{\mu \mu^{\prime}} } \\
& -\sum_{\mu^{\prime \prime}}\left(P_{\mu \mu^{\prime \prime}}^{\mu^{\prime}}-P_{\mu \mu^{\prime}}^{\mu^{\prime \prime}}+P_{\mu^{\prime} \mu^{\prime \prime}}^{\mu}\right)+P_{\mu \mu^{\prime}}^{\mathrm{isoot}} .
\end{aligned}
$$

In the numerical implementation, the Dirac delta conserving the energy is replaced by a Gaussian smearing. As the authors of Ref. [7] noted, the above expression guarantees that the scattering matrix $A$ is symmetric and positivedefinite also in the presence of a Gaussian smearing (other expressions, which would be equivalent to a Dirac delta function, may introduce spurious negative eigenvalues). By virtue of Eqs. (A2) and (A3), it follows that $\tilde{\Omega}$ is symmetric but not $\Omega$, hence the necessity of the transformations (5) and (6).

Finally, we recall that phonon lifetimes are related to the diagonal elements of the scattering matrix as

$$
\begin{aligned}
A_{\mu \mu} & =\frac{\bar{n}_{\mu}\left(\bar{n}_{\mu}+1\right)}{\tau_{\mu}}, \\
\tilde{\Omega}_{\mu \mu} & =\frac{1}{\tau_{\mu}} .
\end{aligned}
$$

\section{APPENDIX B: SILICON THERMAL PROPERTIES}

In this appendix, we study the effect of the scaling of specific heat on velocities and mean free paths. While relaxon properties are as defined in the main text, phonon velocities and mean free paths are scaled as

$$
\begin{aligned}
v_{\mu} & \rightarrow \sqrt{\frac{C_{\mu}}{\mathcal{V} C}} v_{\mu}, \\
\Lambda_{\mu}^{\mathrm{SMA}} & \rightarrow \sqrt{\frac{C_{\mu}}{\mathcal{V} C} \Lambda_{\mu}^{\mathrm{SMA}} .}
\end{aligned}
$$

With this choice of scaling, we can write the SMA thermal conductivity as

$$
\left(k^{i j}\right)^{\mathrm{SMA}}=\sum_{\mu} C v_{\mu}^{i}\left(\Lambda_{\mu}^{j}\right)^{\mathrm{SMA}},
$$

which treats specific heat in the same way as Eq. (13). In Fig. 10, we report the comparison of these scaled phonon quantities with the relaxon properties in silicon. One can readily see that the 2-orders-of-magnitude differences that appeared in Figs. 7 and 8 have almost disappeared. Most of the discrepancy is thus due to the usage of specific heat. Nevertheless, the largest phonon velocities are still a factor of 3 smaller than those of relaxons, and the two pictures do not perfectly overlap.

\section{APPENDIX C: MATTHIESSEN RULE}

Here, we recall a known result [18] that proves that the application of the Matthiessen rule results in an overestimation of the exact thermal conductivity. The BTE for a homogeneous system under a static gradient of temperature can be written in a matrix form (see, for example, Ref. [18] or more recently Ref. [7]):

$$
A \phi=b,
$$

where $A$ is related to the scattering matrix $\Omega$ via $A_{\mu \mu^{\prime}}=\Omega_{\mu \mu^{\prime}} \bar{n}_{\mu^{\prime}}\left(\bar{n}_{\mu^{\prime}}+1\right), b=-\left(\partial \bar{n}_{\mu} / \partial T\right) v_{\mu}$, and $\phi$ is the deviation from equilibrium defined as $n_{\mu}=\bar{n}_{\mu}+$ $\bar{n}_{\mu}\left(\bar{n}_{\mu}+1\right) \nabla T \phi_{\mu}$.

Another way of solving the BTE, besides the diagonalization approach discussed in the main article, is via the variational principle [18]. In particular, the solution of the BTE can be found from the minimization of the functional [18]

$$
\mathcal{F}[\phi]=\frac{\langle\phi|A| \phi\rangle}{(\langle\phi \mid b\rangle)^{2}} .
$$

Let $\phi$ be the function minimizing $\mathcal{F}$. The minimum of $\mathcal{F}$ is directly proportional to the thermal resistivity $\rho$ [18]; therefore, we write

$$
\rho=\frac{1}{k}=\mathcal{F}[\phi]
$$




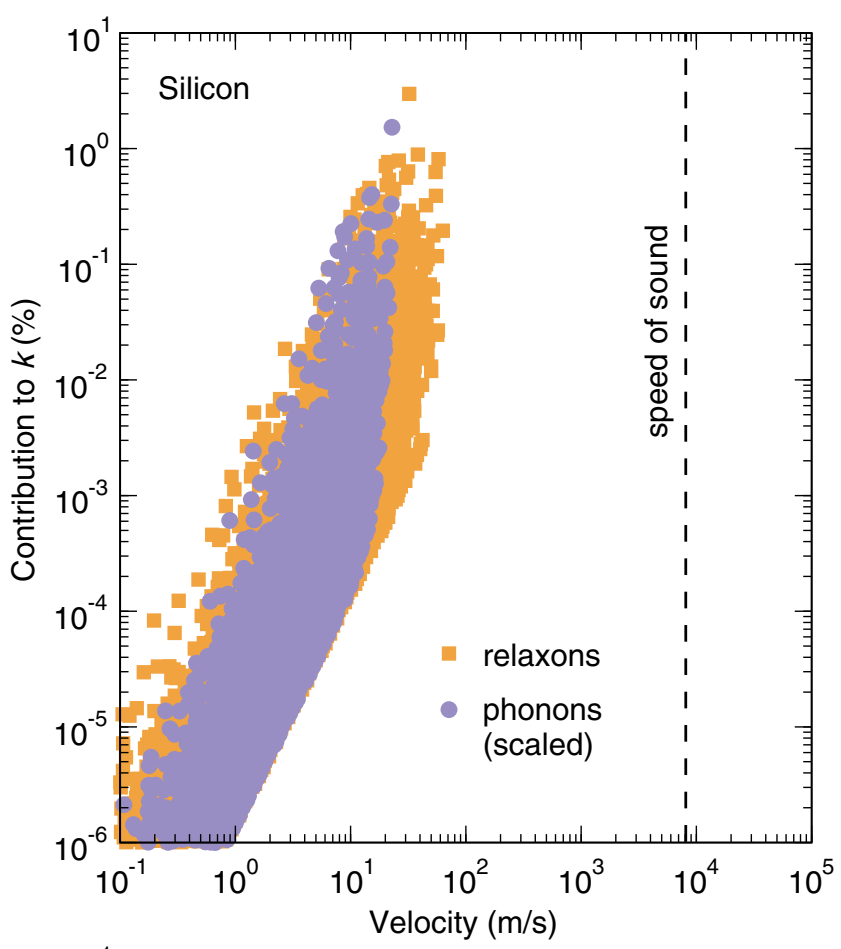

The exact resistivity is given by

$$
\rho=\frac{\left\langle\phi\left|A_{1}\right| \phi\right\rangle+\left\langle\phi\left|A_{2}\right| \phi\right\rangle}{(\langle\phi \mid b\rangle)^{2}} .
$$

The function $\phi$ that minimizes the functional defined by $A$ will not, in general, be the function that minimizes the functionals $\mathcal{F}_{1}$ and $\mathcal{F}_{2}$ defined by $A_{1}$ or $A_{2}$ only. The functionals $\mathcal{F}_{1}$ and $\mathcal{F}_{2}$ instead will be minimized by the functions $\phi_{1}$ and $\phi_{2}$, respectively. By the variational principle,

$$
\begin{aligned}
\rho & =\frac{\left\langle\phi\left|A_{1}\right| \phi\right\rangle}{(\langle\phi \mid b\rangle)^{2}}+\frac{\left\langle\phi\left|A_{2}\right| \phi\right\rangle}{(\langle\phi \mid b\rangle)^{2}} \\
& \geq \frac{\left\langle\phi_{1}\left|A_{1}\right| \phi_{1}\right\rangle}{\left(\left\langle\phi_{1} \mid b\right\rangle\right)^{2}}+\frac{\left\langle\phi_{2}\left|A_{2}\right| \phi_{2}\right\rangle}{\left(\left\langle\phi_{2} \mid b\right\rangle\right)^{2}} \\
& =\rho_{1}+\rho_{2} .
\end{aligned}
$$

Alternatively, this can be written as

$$
\frac{1}{k} \leq \frac{1}{k_{1}}+\frac{1}{k_{2}}
$$

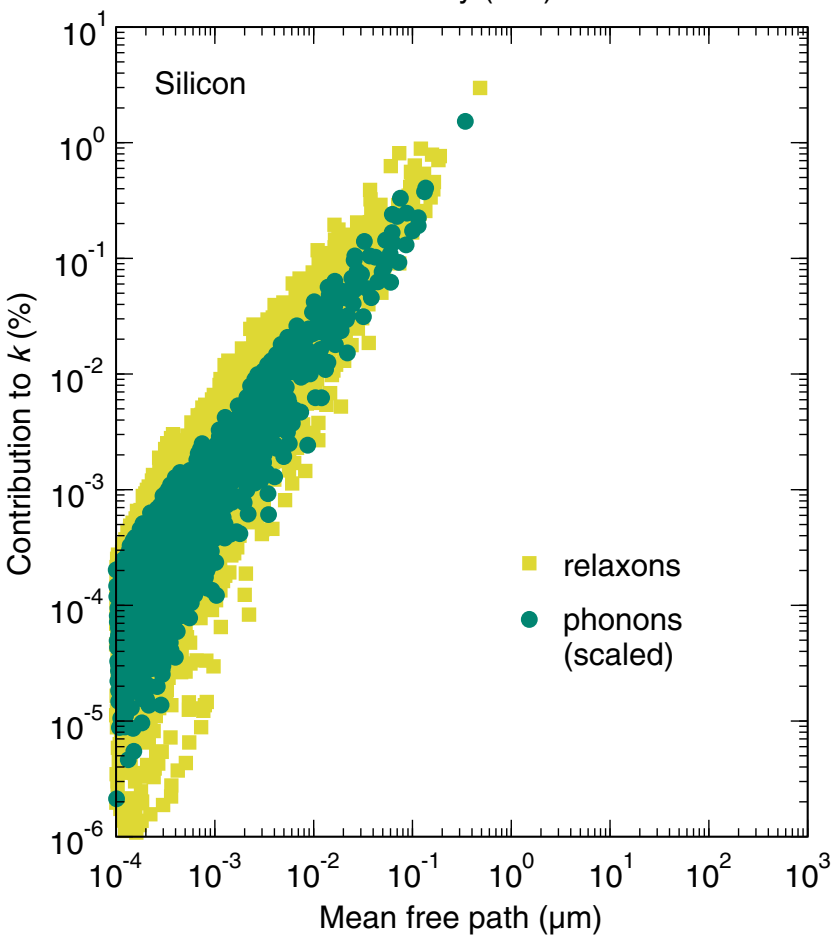

FIG. 10. Top panel: Comparison of relaxon velocities with scaled phonon velocities. Bottom panel: Same as the top panel but for mean free paths.

Now, let us separate the scattering matrix into two different components (for example, three-phonon and isotopic scattering),

$$
A=A_{1}+A_{2} \text {. }
$$

showing that the Matthiessen rule is a special case where the equalities hold exactly. More generally, its application leads to an overestimation of thermal conductivities.

\section{APPENDIX D: ITERATIVE METHOD}

In this appendix, we examine the convergence properties of the iterative method for solving the BTE. Such a method can be formalized as follows. The steady-state homogeneous BTE in the phonon basis is

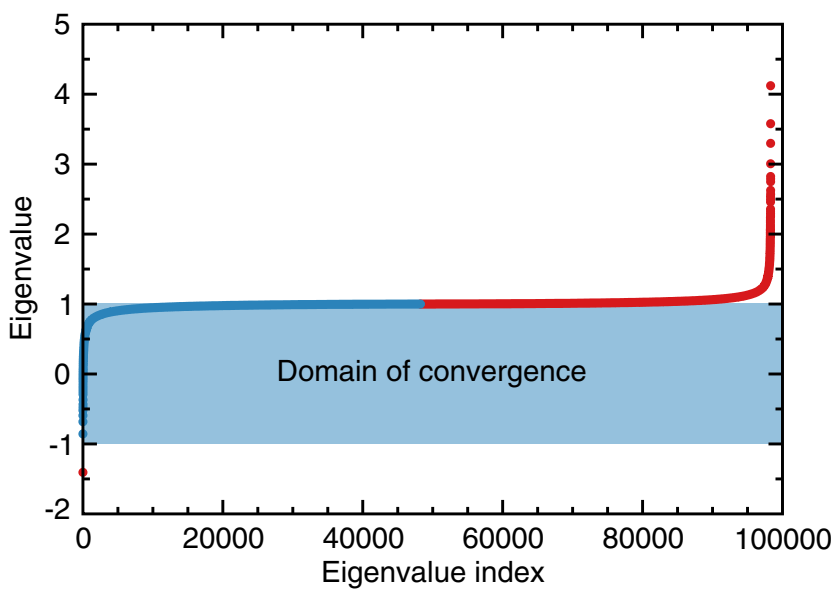

FIG. 11. Eigenvalues $\lambda$ of the matrix $A_{\mathrm{d}}^{-1} A_{\mathrm{od}}$ (see main text for the definition), for graphene at room temperature, ordered by their magnitude. The red dots, roughly half of the eigenvalue spectrum, indicate eigenvalues $|\lambda|>1$ that cause a divergence of the iterative solution for the Boltzmann transport equation. Most of the unstable eigenvalues are greater than 1 , with only one eigenvalue lower than -1 . 


$$
\nabla T \cdot v_{\mu} \frac{\partial \bar{n}_{\mu}}{\partial T}=-\frac{1}{\mathcal{V}} \sum_{\mu^{\prime}} \Omega_{\mu \mu^{\prime}} \Delta n_{\mu^{\prime}}
$$

This is a linear algebra problem of the form $A F=b$, where $b_{\mu}=-v_{\mu} \hbar \omega_{\mu} \bar{n}_{\mu}\left(\bar{n}_{\mu}+1\right) / k_{B} T^{2}, F$ is defined by $n_{\mu}=\bar{n}_{\mu}+\bar{n}_{\mu}\left(\bar{n}_{\mu}+1\right) \nabla T F_{\mu}$, and $\Omega_{\mu \mu^{\prime}}=A_{\mu \mu^{\prime}} \bar{n}_{\mu^{\prime}}\left(\bar{n}_{\mu^{\prime}}+1\right)$. The iterative solution for $F[4,16,17]$ can then be recast [7] as a geometric series $F=\sum_{j=0}^{\infty}\left[-\left(A^{d}\right)^{-1} A^{o d}\right]^{j}\left(A^{d}\right)^{-1} b$, where $A^{d}$ and $A^{o d}$ are, respectively, the diagonal and the off-diagonal parts of $A$. This series is convergent if and only if all the eigenvalues $\lambda$ of $\left[\left(A^{d}\right)^{-1} A^{o d}\right]$ are $|\lambda|<1$. In Fig. 11, we show that, in graphene, $|\lambda|>1$ for more than half of the spectrum, proving that the iterative method is numerically unstable for graphene at room temperature. In general, one might expect convergence issues for the iterative method whenever the relaxon picture differs significantly from the phonon picture and the contribution of the off-diagonal part is large compared to the diagonal part.

[1] R. Peierls, Zur Kinetischen Theorie der Wärmeleitung in Kristallen, Ann. Phys. (N.Y.) 395, 1055 (1929).

[2] M. S. Green, Markoff Random Processes and the Statistical Mechanics of Time-Dependent Phenomena. II. Irreversible Processes in Fluids, J. Chem. Phys. 22, 398 (1954).

[3] R. Kubo, Statistical-Mechanical Theory of Irreversible Processes. I. General Theory and Simple Applications to Magnetic and Conduction Problems, J. Phys. Soc. Jpn. 12, 570 (1957).

[4] D. A. Broido, A. Ward, and N. Mingo, Lattice Thermal Conductivity of Silicon from Empirical Interatomic Potentials, Phys. Rev. B 72, 014308 (2005).

[5] D. A. Broido, M. Malorny, G. Birner, N. Mingo, and D. A. Stewart, Intrinsic Lattice Thermal Conductivity of Semiconductors from First Principles, Appl. Phys. Lett. 91, 231922 (2007).

[6] J. Garg, N. Bonini, B. Kozinsky, and N. Marzari, Role of Disorder and Anharmonicity in the Thermal Conductivity of Silicon-Germanium Alloys: A First-Principles Study, Phys. Rev. Lett. 106, 045901 (2011).

[7] G. Fugallo, M. Lazzeri, L. Paulatto, and F. Mauri, Ab Initio Variational Approach for Evaluating Lattice Thermal Conductivity, Phys. Rev. B 88, 045430 (2013).

[8] K. Esfarjani, G. Chen, and H. T. Stokes, Heat Transport in Silicon from First-Principles Calculations, Phys. Rev. B 84, 085204 (2011).

[9] A. Ward, D. A. Broido, D. A. Stewart, and G. Deinzer, $A b$ Initio Theory of the Lattice Thermal Conductivity in Diamond, Phys. Rev. B 80, 125203 (2009).

[10] M. N. Luckyanova, J. Garg, K. Esfarjani, A. Jandl, M. T. Bulsara, A. J. Schmidt, A. J. Minnich, S. Chen, M. S. Dresselhaus, Z. Ren, E. A. Fitzgerald, and G. Chen, Coherent Phonon Heat Conduction in Superlattices, Science 338, 936 (2012).

[11] D. G. Cahill, P. V. Braun, G. Chen, D. R. Clarke, S. Fan, K. E. Goodson, P. Keblinski, W. P. King, G. D. Mahan,
A. Majumdar et al., Nanoscale Thermal Transport. II. 2003-2012, Appl. Phys. Rev. 1, 011305 (2014).

[12] D. Donadio and G. Galli, Atomistic Simulations of Heat Transport in Silicon Nanowires, Phys. Rev. Lett. 102, 195901 (2009).

[13] A. J. H. McGaughey and M. Kaviany, Quantitative Validation of the Boltzmann Transport Equation Phonon Thermal Conductivity Model under the Single-Mode Relaxation Time Approximation, Phys. Rev. B 69, 094303 (2004).

[14] S. G. Volz and G. Chen, Molecular Dynamics Simulation of Thermal Conductivity of Silicon Nanowires, Appl. Phys. Lett. 75, 2056 (1999).

[15] G. Zhang and B. Li, Thermal Conductivity of Nanotubes Revisited: Effects of Chirality, Isotope Impurity, Tube Length, and Temperature, J. Chem. Phys. 123, 114714 (2005).

[16] M. Omini and A. Sparavigna, Heat Transport in Dielectric Solids with Diamond Structure, Nuovo Cimento D 19, 1537 (1997).

[17] M. Omini and A. Sparavigna, Beyond the Isotropic-Model Approximation in the Theory of Thermal Conductivity, Phys. Rev. B 53, 9064 (1996).

[18] J. Ziman, Electrons and Phonons: The Theory of Transport Phenomena in Solids, Oxford Classic Texts in the Physical Sciences (Oxford University Press, New York, 2001).

[19] R. A. H. Hamilton and J. E. Parrot, Variational Calculation of the Thermal Conductivity of Germanium, Phys. Rev. 178, 1284 (1969).

[20] G. P. Srivastava, Derivation and Calculation of Complementary Variational Principles for the Lattice Thermal Conductivity, J. Phys. C 9, 3037 (1976).

[21] R. A. Guyer and J. A. Krumhansl, Solution of the Linearized Phonon Boltzmann Equation, Phys. Rev. 148, 766 (1966).

[22] R. J. Hardy, Phonon Boltzmann Equation and Second Sound in Solids, Phys. Rev. B 2, 1193 (1970).

[23] L. Chaput, Direct Solution to the Linearized Phonon Boltzmann Equation, Phys. Rev. Lett. 110, 265506 (2013).

[24] J. Callaway, Model for Lattice Thermal Conductivity at Low Temperatures, Phys. Rev. 113, 1046 (1959).

[25] L. Lindsay, D. A. Broido, and N. Mingo, Flexural Phonons and Thermal Transport in Graphene, Phys. Rev. B 82, 115427 (2010).

[26] A. Cepellotti, G. Fugallo, L. Paulatto, M. Lazzeri, F. Mauri, and N. Marzari, Phonon Hydrodynamics in TwoDimensional Materials, Nat. Commun. 6, 6400 (2015).

[27] L. Lindsay and D. A. Broido, Enhanced Thermal Conductivity and Isotope Effect in Single-Layer Hexagonal Boron Nitride, Phys. Rev. B 84, 155421 (2011).

[28] A. Jain and A.J.H. McGaughey, Strongly Anisotropic In-Plane Thermal Transport in Single-Layer Black Phosphorene, Sci. Rep. 5, 8501 (2015).

[29] G. Fugallo, A. Cepellotti, L. Paulatto, M. Lazzeri, N. Marzari, and F. Mauri, Thermal Conductivity of Graphene and Graphite: Collective Excitations and Mean Free Paths, Nano Lett. 14, 6109 (2014).

[30] M. Gill-Comeau and L. J. Lewis, On the Importance of Collective Excitations for Thermal Transport in Graphene, Appl. Phys. Lett. 106, 193104 (2015). 
[31] S. Lee, D. Broido, K. Esfarjani, and G. Chen, Hydrodynamic Phonon Transport in Suspended Graphene, Nat. Commun. 6, 6290 (2015).

[32] G. Barbarino, C. Melis, and L. Colombo, Intrinsic Thermal Conductivity in Monolayer Graphene Is Ultimately Upper Limited: A Direct Estimation by Atomistic Simulations, Phys. Rev. B 91, 035416 (2015).

[33] C. de Tomas, A. Cantarero, A. F. Lopeandia, and F. X. Alvarez, From Kinetic to Collective Behavior in Thermal Transport on Semiconductors and Semiconductor Nanostructures, J. Appl. Phys. 115, 164314 (2014).

[34] R. J. Hardy, Energy-Flux Operator for a Lattice, Phys. Rev. 132, 168 (1963).

[35] R. J. Hardy, Lowest-Order Contribution to the Lattice Thermal Conductivity, J. Math. Phys. (N.Y.) 6, 1749 (1965).

[36] J. A. Krumhansl, Thermal Conductivity of Insulating Crystals in the Presence of Normal Processes, Proc. Phys. Soc. 85, 921 (1965).

[37] A. A. Balandin, S. Ghosh, W. Bao, I. Calizo, D. Teweldebrhan, F. Miao, and C. N. Lau, Superior Thermal Conductivity of Single-Layer Graphene, Nano Lett. 8, 902 (2008).

[38] S. Baroni, S. de Gironcoli, A. Dal Corso, and P. Giannozzi, Phonons and Related Crystal Properties from DensityFunctional Perturbation Theory, Rev. Mod. Phys. 73, 515 (2001).

[39] P. Giannozzi, S. de Gironcoli, P. Pavone, and S. Baroni, Ab Initio Calculation of Phonon Dispersions in Semiconductors, Phys. Rev. B 43, 7231 (1991).

[40] A. Debernardi, S. Baroni, and E. Molinari, Anharmonic Phonon Lifetimes in Semiconductors from DensityFunctional Perturbation Theory, Phys. Rev. Lett. 75, 1819 (1995).

[41] L. Paulatto, F. Mauri, and M. Lazzeri, Anharmonic Properties from a Generalized Third-Order Ab Initio Approach: Theory and Applications to Graphite and Graphene, Phys. Rev. B 87, 214303 (2013).

[42] M. Lazzeri and S. de Gironcoli, First-Principles Study of the Thermal Expansion of Be(1010), Phys. Rev. B 65, 245402 (2002).

[43] S. Baroni, P. Giannozzi, and A. Testa, Green's-Function Approach to Linear Response in Solids, Phys. Rev. Lett. 58, 1861 (1987).

[44] X. Gonze and J.-P. Vigneron, Density-Functional Approach to Nonlinear-Response Coefficients of Solids, Phys. Rev. B 39, 13120 (1989).

[45] P. Giannozzi, S. Baroni, N. Bonini, M. Calandra, R. Car, C. Cavazzoni, D. Ceresoli, G. L. Chiarotti, M. Cococcioni, I. Dabo et al., QUANTUM ESPRESSO: A Modular and
Open-Source Software Project for Quantum Simulations of Materials, J. Phys. Condens. Matter 21, 395502 (2009).

[46] N. Bonini, J. Garg, and N. Marzari, Acoustic Phonon Lifetimes and Thermal Transport in Free-Standing and Strained Graphene, Nano Lett. 12, 2673 (2012).

[47] Y. Kuang, L. Lindsay, and B. Huang, Unusual Enhancement in Intrinsic Thermal Conductivity of Multilayer Graphene by Tensile Strains, Nano Lett. 15, 6121 (2015).

[48] J. Garg, N. Bonini, and N. Marzari, First-Principles Determination of Phonon Lifetimes, Mean Free Paths, and Thermal Conductivities in Crystalline Materials: Pure Silicon and Germanium, in Length-Scale Dependent Phonon Interactions Topics in Applied Physics Vol. 128, edited by S. L. Shind and G. P. Srivastava, Length-Scale Dependent Phonon Interactions (Springer, New York, 2014).

[49] P. Jiang, L. Lindsay, and Y. K. Koh, Role of Low-Energy Phonons with Mean-Free-Paths $>0.8 \mu \mathrm{m}$ in Heat Conduction in Silicon, J. Appl. Phys. 119, 245705 (2016).

[50] J. Cuffe, J. K. Eliason, A. A. Maznev, K. C. Collins, J. A. Johnson, A. Shchepetov, M. Prunnila, J. Ahopelto, C. M. Sotomayor Torres, G. Chen, and K. A. Nelson, Reconstructing Phonon Mean-Free-Path Contributions to Thermal Conductivity Using Nanoscale Membranes, Phys. Rev. B 91, 245423 (2015).

[51] A. J. Minnich, J. A. Johnson, A. J. Schmidt, K. Esfarjani, M. S. Dresselhaus, K. A. Nelson, and G. Chen, Thermal Conductivity Spectroscopy Technique to Measure Phonon Mean Free Paths, Phys. Rev. Lett. 107, 095901 (2011).

[52] K. T. Regner, D. P. Sellan, Z. Su, C. H. Amon, A. J. H. McGaughey, and J.A. Malen, Broadband Phonon Mean Free Path Contributions to Thermal Conductivity Measured Using Frequency Domain Thermoreflectance, Nat. Commun. 4, 1640 (2013).

[53] A. Dal Corso, PsLibrary, http://qe-forge.org/gf/project/ pslibrary (2012).

[54] M. E. Wieser, N. Holden, T. B. Coplen, J. K. Boehlke, M. Berglund, W. A. Brand, P. De Bièvre, M. Groening, R. D. Loss, J. Meija et al., Atomic Weights of the Elements 2011 (IUPAC Technical Report), Pure Appl. Chem. 85, 883 (2013).

[55] L. S. Blackford, J. Choi, A. Cleary, E. D’Azevedo, J. Demmel, I. Dhillon, J. Dongarra, S. Hammarling, G. Henry, A. Petitet et al., ScaLAPACK Users' Guide (Society for Industrial and Applied Mathematics, Philadelphia, PA, 1997).

[56] G. Pizzi, A. Cepellotti, R. Sabatini, N. Marzari, and B. Kozinsky, AiiDA: Automated Interactive Infrastructure and Database for Computational Science, Comput. Mater. Sci. 111, 218 (2016). 